\title{
In Vitro Colony Formation of Carp Granulocytes in a Semi-Solid Agar Culture System
}

\author{
Tadaaki Moritomo*, Akihide Itou*, Itaru Yamaguchi** \\ and Tasuku Watanabe* \\ *Laboratory of Fish Pathology, Department of Veterinary Science, Nihon University, \\ Kameino, Fujisawa 252, Japan \\ ** Department of Biological Sciences, Central Research Laboratories, Zeria Pharmaceutical Co., LTD., \\ Oshikiri, Konan, Osato, Saitama 360-01, Japan
}

(Received November 16, 1992)

\begin{abstract}
Carp (Cyprinus carpio) hematopoietic cells collected from the kidney were cultured in vitro in 35 mm-diameter tissue culture dishes using $1 \mathrm{ml}$ of RPMI-1640 medium, $20 \%$ fetal calf serum, varying concentrations of carp serum and $0.3 \%$ agarose. The cells at a concentration of $2 \times 10^{5}$ cells $/ \mathrm{ml}$ in agar-medium were incubated for $7-10$ days at $30^{\circ} \mathrm{C}$ with $5 \% \mathrm{CO}_{2}$.

Many small aggregates constituting 3 to 10 cells were observed 3 or 4 days after incubation. Most aggregates grew and formed colonies which were made up of 50 to 100 cells. In contrast, when cultured without carp serum, most of the cells did not multiply and disappeared by day 7. To investigate the colony-forming cell types, agar cultures were dried and stained with May-Grünwald Giemsa. It was found that the colonies containd blast cells, of granulocytes several developmental stages and mature granulocytes. The mature cells had a weakly acidophilic or chromophoric cytoplasm and a single oval or kidney shaped nucleus, and were positive for peroxidase reaction. Electron microscopy showed many granules containing electron-dense materials in the cytoplasm of the matured cells.
\end{abstract}

The importance of granulocytes in host defense mechanisms in fish is well demonstrated (Ellis, 1977; Rowley et al., 1988), but little is known about the mechanisms which regulate multiplication and differentiation of such cells. A semi-solid agar culture technique for human and murine bone marrow cells was introduced to investigate the process of hematopoiesis in vitro (Plunznik and Sachs, 1966; Bradley and Metcalf, 1966). This culture system permits the progenitors of granulocytes and/or macrophages to grow and form colonies when stimulated with particular glycoproteins, such as colony stimulating factors (CSFs). The CSFs have been found from various sources including sera, urine, and feeder layers from various tissues or media conditioned by these tissues (Metcalf, 1986). By using this culture system, several different CSFs of human or murine origin have been identified and well characterized (Steven and Kamen, 1987; Metcalf, 1989).

In the present study, we developed a semi-solid culture system for the generation of hemtopietic colonies from carp kidney cells. This new system may be useful in the determination and characterization of the factors crucial to carp granulocyte development.

\section{Materials and Methods}

Preparation of Carp Serum and Kidney Cell Suspensions

Six to ten-months old carp, Cyprinus carpio (weighing 10-25g) were obtained from a commercial farm and kept in a tank at $22-25^{\circ} \mathrm{C}$. Blood was drawn from the carp, and serum was separated by a centrifugation after the blood was allowed to clot at $4^{\circ} \mathrm{C}$ for 2 hours. The sera from more than 10 carp were pooled, sterilized through a Millipore filter $(0.45 \mu \mathrm{m})$, and frozen at $-20^{\circ} \mathrm{C}$ until used.

Carp were anesthetized by immersion in 1:20000 (w/v) tricaine methanesulfonate (MS-222). Whole kidney was dissected from the carp and macerated on a stainless steel mesh with two forceps in RPMI1640 medium to release kidney hematopietic cells. The cells were collected by cetrifugation $(200 \times g, 5$ 
min.) and $5 \mathrm{ml}$ of distilled water was added to cell pellet to lyse mature erythrocytes. After $10 \mathrm{~s}, 5 \mathrm{ml}$ of $2 \times$ RPMI-1640 was added to the cells and the cells were washed twice by centrifugation with RPMI1640. The cells were dispersed in RPMI-1640 by vigorous pipetting and adjusted to a concentration of $1-1.5 \times 10^{6} \mathrm{cell} / \mathrm{ml}$.

\section{Culture Method}

A culture technique modified from the methods of Metcalf (1970) for mouse bone marrow cells was employed. Briefly, $0.2 \mathrm{ml}$ of carp kidney cell suspension, $0.1 \mathrm{~m} l$ of carp serum diluted in RPMI-1640 and $0.2 \mathrm{ml}$ of fetal calf serum were added to $35 \mathrm{~mm}$ diameter tissue culture dishes. To this mixture was then added $0.5 \mathrm{ml}$ of $0.6 \%$ agarose (SeaKem LE agarose, FMC Bioproducts, ME, USA) in RPMI1640 which had been prewarmed at $42^{\circ} \mathrm{C}$. Then, the dishes were shaken gently and cooled at $4{ }^{\circ} \mathrm{C}$. Control dishes were prepared in all experiments in the same manner except for the addition of RPMI-1640 instead of carp serum. The dishes were incubated for 5 to 10 days under $5 \% \mathrm{CO}_{2}-95 \%$ air at $30^{\circ} \mathrm{C}$.

To investigate the effects of the carp serum on colony formation, 4 fold serial dilutions of the pooled carp serum $(1: 4$ to $1: 1024)$ were prepared in RPMI-1640 and $0.1 \mathrm{ml}$ of each dilution of carp serum was added to the culture as described previously.

\section{Light Microscopy}

After incubation for 5-7 days, the agar cultures were prepared for light microscopic examinations. One or two filter papers were placed on the agar surface to absorb the moisture. The agar cultures were then dried by blowing cold air using a hair dryer. The resulting agar film was stained with May-Grünwald Giemsa. Peroxidase staining was also performed.

\section{Electron Microscopy}

The surface of the agar was covered with $2 \%$ glutaraldehyde in $0.2 \mathrm{M}$ phosphate buffer (PB, pH 7.4) for $2 \mathrm{~h}$ at $4^{\circ} \mathrm{C}$. After washing 3 times with $\mathrm{PB}$, colonies in the agar were aspirated, fixed with $1 \%$ $\mathrm{OsO}_{4}$, dehydrated in a graded ethanol series and embedded in Quetol-812 (Nisshin EM Co., Tokyo, Japan). Ultrathin sections were double-stained with uranyl acetate and lead citrate, and examined with a JEM1200 electron microscope.

\section{Results}

\section{Colony Formation}

When the kindney hematopoietic cells were cultured in the agar medium containing carp serum, small aggregates of 3 to 10 cells were first observed in several parts of the dishes on days 3 and 4 after initiation. The number of aggregates progressively increased with time. Some grew rapidly and formed colonies which were made up of 50 to 100 cells. Most of the colonies were constituted of round or oval-shaped cells which were tightly aggregated (Fig. 1). A small number of the colonies were composed of ameboid cells which were not aggregated and spreaded into the surrounding agar (Fig. 2).

Table 1 shows the effect of carp serum supplementation on colony formation. Ten cell suspensions were preparared from the kidney of 10 carp. When the cells were incubated with $2.5 \%$ carp serum, significant increase in number was observed in the colony counts. However, the number of colonies varied among individual carp, the number was always higher than that when carp serum was not used. Most of the cells in control cultures did not multiply and had disapperared by day 7 , although a few persistent colonies were observed in 3 dishes.

A dose-response relationship was observed between the amount of carp serum and the number of colonies (Table 2). The umber of colonies decreased with the dilution of carp serum in each case. The number of colonies was often too numerous to count at 1:4 dilution of carp serum. However 1:256 dilution of carp serum still showed some stimulatory effect on the growth of colonies.

\section{Morphology of Colony Forming Cells}

Several types of cells were observed in a colony stained by May-Grünwald Giemsa. Figs. 3 and 4 show colonies on day 7 in a culture supplemented with carp serum. Most of the cells had a granulocyte-like appearance. They were round or oval-shaped, and had weakly acidophilic or chromophoric cytoplasm. The nucleus was oval to kidney-shaped. Occasionally, blastic cells with basophilic cytoplasm and a round nuclus were observed in those colonies. By peroxidase staining, some cells with an oval or kidney-shaped nucleus were positive for peroxidase, while immature blast cells remained negative for peroxidase. 


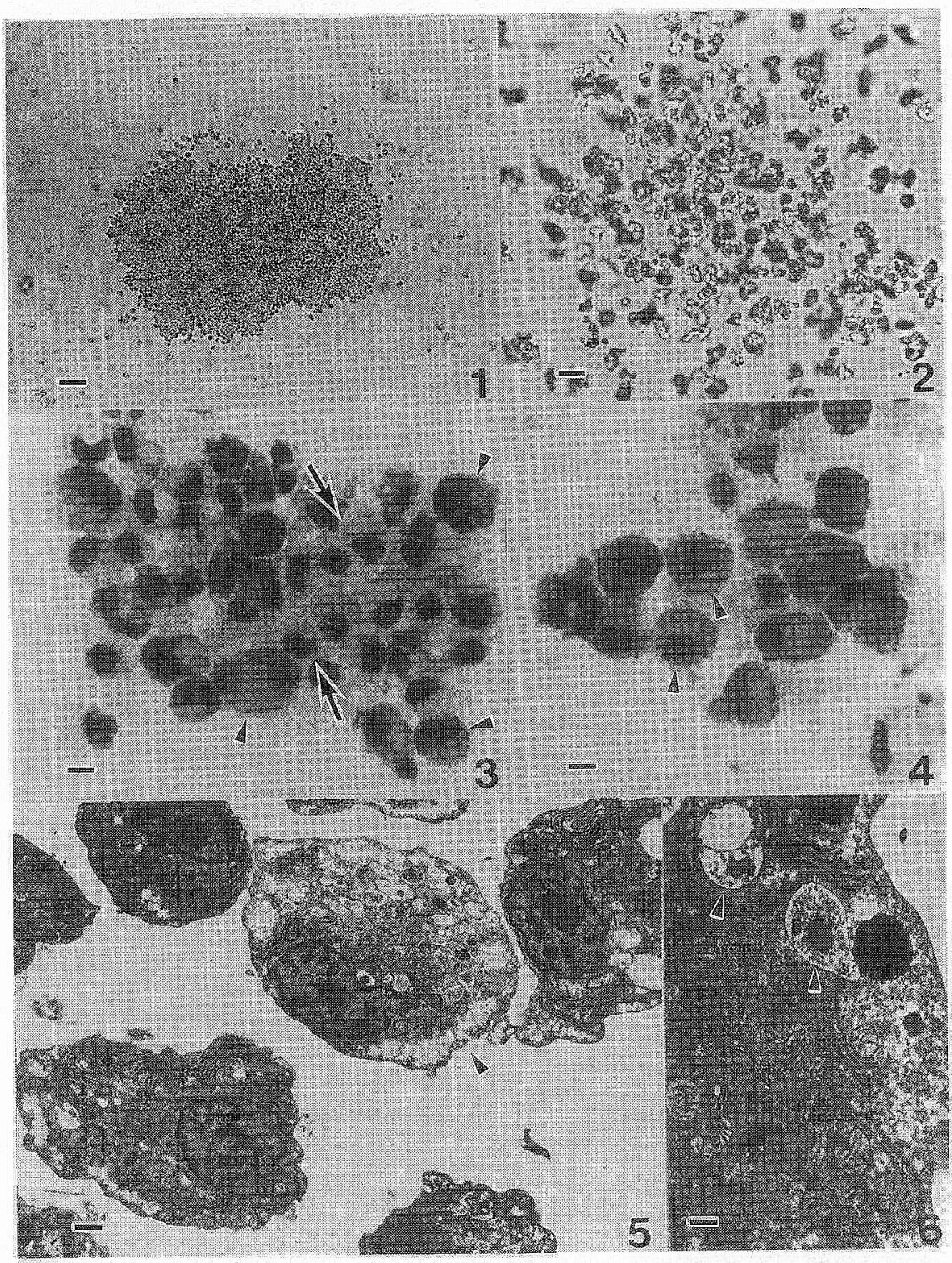

Fig. 1. A typical colony of carp granulocytes 7 days after initiation. Cells was tightly aggregated. Scale: 100 $\mu \mathrm{m}$.

Fig. 2. A colony of ameboid cells. This second cell type did not aggregate, and spread into the surrounding agar. $20 \mu \mathrm{m}$.

Fig. 3. A portion of a colony in soft agar showing two cell types. One cell type has characteristics of a mature granulocyte with a kidney-shaped nucleus and weakly acidophilic or ehromophoric eytoplasm (full arrows). The other has a round or oval nucleus. These cells has a basophilic cytoplasm (arrowheads). May Grünwald-Giemsa stain. Scale: $10 \mu \mathrm{m}$.

Fig. 4. A portion of a colony in soft agar after 7 days of culture. The majority of the cells showed immature characteristics, with large, round in shape and basophilic cytoplasm. Some cells in this colony were mature with a small, oval nuclus (arrowheads). May Grünwald-Giemsa stain. Scale: $10 \mu \mathrm{m}$.

Fig. 5. An electron micrograph showing cells from a colony in soft agar. The cells have many mitochondria and a well developed rough-surfaced endoplasmic reticulum. One of the cells (arrow head) has cytoplasmic granules containing an electron-dense material. Scale: $1.2 \mu \mathrm{m}$.

Fig. 6. An electron micrograph showing a portion of a cell from a colony grown in soft agar. Granules containing electron-dense material are shown in the cytoplasm (arrow heads). Scale: $375 \mathrm{~nm}$. 
Electron microscopic examination revealed that most of the cells were round to oval and possessed an eccentric nucleus with a prominent uncleolus. They had many mitochondria, well developed roughsurfaced endoplasmic reticulum, and Golgi apparatus. Some cells had many variously sized $(0.5-1 \mu \mathrm{m})$ granules containing electron dense material (Fig. 5). The granules were distributed in the cytoplasm and had a dark content with patchy appearing light zones. Some granules filled with very dense material were also observed in the cytoplasm (Fig. 6).

\section{Discussion}

It was possible to grow carp hematopoietic cells in a semi-solid agar medium. The cells can multiply

Table 1. Effect of carp serum supplementation on colony formation

\begin{tabular}{ccc}
\hline \hline \multirow{2}{*}{$\begin{array}{c}\text { Cell suspension } \\
\text { number* }\end{array}$} & \multicolumn{2}{c}{ Number of colonies formed } \\
\cline { 2 - 3 } & Carp serum** & Control \\
\hline 1 & $55, \quad 55$ & 5,0 \\
2 & 40,2 & 0,0 \\
3 & $8, \quad 63$ & 1,0 \\
4 & 27,131 & 0,0 \\
5 & 38,31 & 0,0 \\
6 & 39,35 & 0,0 \\
7 & 15,87 & 0,0 \\
8 & 12,21 & 0,0 \\
9 & $15, \quad 11$ & 0,0 \\
10 & $9, \quad 9$ & 0,1 \\
\hline
\end{tabular}

* Ten cell suspensions were prepared from the kidneys of 10 carp.

** Each culture contained $0.1 \mathrm{~m} l$ of $1: 4$ carp serum and $2-3 \times 10^{5}$ carp cells. Colony counts were performed on duplicate dishes. quickly and form colonies in the presence of carp serum. The colonies contained several morphological or developmental stages of granulocytes i.e., blast cells to mature granulocytes. The mature cells had an oval or kidney-shaped nucleus, and were positive for peroxidase. Electronmicroscopically, the mature cells had many granules resembling the granules of carp granulocytes in the peripheral blood (Rowly et al., 1988). These morphological appearances of the colony cells indicate that a certain cell in hematopoietic tissue multiplies in agar-media and forms a colony composed of different developmental stages of the granulocytes, and suggest that colony cells initiated from a progenitor cell of granulocytes lineage proliferate and differentiate into mature granulocytes in the agar medium.

In mammals, clonal growth of bone marrow cells in soft gel culture is largely dependent on colony stimulating factors (CSEs). These factors, derived from human and mouse serum, are well characterized. Injection of endotoxin into mice is known to cause a high level of serum colony stimulating activity (Metcalf, 1971). Human serum or plasma is also capable of stimulating colony formation from human marrow cells (Metcalf, 1974). In the present study, significant increse in the number of colonies was seen in the presence of carp serum. Furthermore, colony formation was dependent upon the concentration of carp serum. These results indicate that the carp serum enhances the formation of carp granulocyte colonies in semi-solid agar.

Although it is still not certain whether carp have CSF regulatory mechanisms similar to mammals, the present cultue system will be useful in resolving this question and in exploring the ontogeny of carp granulocytes.

Table 2. Effect of carp serum dilution on the number of colonies formed

\begin{tabular}{|c|c|c|c|c|c|c|}
\hline \multirow{2}{*}{$\begin{array}{c}\text { Expt. } \\
\text { No. }\end{array}$} & \multicolumn{6}{|c|}{ Serum dilution } \\
\hline & $1: 4$ & $1: 16$ & $1: 64$ & $1: 256$ & $1: 1024$ & Control \\
\hline \multirow[t]{2}{*}{1} & 78 & 52 & 10 & 0 & 4 & 0 \\
\hline & 76 & 24 & 7 & 9 & 2 & 1 \\
\hline \multirow[t]{2}{*}{2} & T.N.T.C.* & 110 & 61 & 83 & 26 & 4 \\
\hline & T.N.T.C. & 130 & 65 & 62 & 37 & 1 \\
\hline \multirow[t]{2}{*}{3} & T.N.T.C. & T.N.T.C. & 189 & 23 & 4 & 0 \\
\hline & T.N.T.C. & T.N.T.C. & 134 & 19 & 1 & 5 \\
\hline
\end{tabular}

Each culture contained diluted carp serum in serial dilutions of $1: 4$ to $1: 1024$. Colony counts were performed on duplicate dishes.

* T.N.T.C.: Too numerous to count. 


\section{Acknowledgements}

The authors wish to thank Dr. D. P. Aderson, U. S. Fish and Wildlife Service, National Fish Health Research Laboratory, for his professional advice and encouragement and $T$. Imagawa, Department of Veterinary Anatomy, Faculty of Veterinary Medicine, Hokkaido University, for his counsel on histological matters.

This study was granted by Grant-in-Aid for Scientific Research (C) and Grant-in-Aid for Encouragement of Young Scientists from the Ministry of Education, Science and Culture, Japanes Governments.

\section{References}

Bradley, T. R. and D. Metcalf (1966): The growth of mouse bone marrow cells in vitro. Aust. J. Exp. Biol. Med. Sci., 44, 287-300.

Ellis, A. E. (1977): The leukocytes of fish. A review. $J$. Fish Biol., 11, 453-491.

Metcalf, D. (1970): Studies on colony formation in vitro by mouse bone marrow cells. II. Action of colony stimulating factor. J. Cell. Physiol., 76, 89-100.

Metcalf, D. (1971): Acute antigen-induced elevation of serum colony stimulating factor (CSF) levels. Immunology, 21, 427-436.

Metcalf, D. (1974): Stimulation by human urine or plasma of granulopoiesis by human marrow cells in agar. Exp. Hematol., 2, 157-173.

Metcalf, D. (1986): The molecular biology and function of the granulocyte-macrophage colony-stimulating factors. Blood, 67, 257-267.

Metcalf, D. (1989): The molecular control of cell division, differentiation commitment and maturation in haemopoietic cells. Nature, 339 27-30.

Plunznik, D. H. and L. Sachs (1966): The cloning of normal "mast" cells in tissue culture. J. Cell. Physiol., 66, 319-324.

Rowley, A. F., T. C. Hunt, M. Page and G. Mainwaring (1988): Fish. In "Vertebrate blood cells" (ed. by A. F. Rowley and N. A. Ratcliffe). Cambridge University Press, pp. 19-127.

Steven, C. C. and R. Kamen (1987): The human hematopoietic colony-stimulating factors. Nature, 236, 1229 1236. 